\title{
The Point Charge Oscillator: Qualitative and Analytical Investigations
}

\section{Klaus R. Schneider}

Weierstrass Institute for Applied Analysis and Stochastics

Mohrenstr. 39, 10117 Berlin

E-mail(corresp.): schneider@wias-berlin.de

Received September 7, 2018; revised April 1, 2019; accepted April 2, 2019

\begin{abstract}
We study the mathematical model of the point charge oscillator which has been derived by A. Beléndez et al. [2]. First we determine the global phase portrait of this model in the Poincaré disk. It consists of a family of closed orbits surrounding the unique finite equilibrium point and of a continuum of homoclinic orbits to the unique equilibrium point at infinity.

Next we derive analytic expressions for the relationship between period (frequency) and amplitude. Further, we prove that the period increases monotone with the amplitude and derive an expression for its growth rate as the amplitude tends to infinity. Finally, we determine a relation between period and amplitude by means of the complete elliptic integral of the first kind $K(k)$ and of the Jacobi elliptic function $\mathrm{cn}$.
\end{abstract}

Keywords: point charge oscillator, global phase portrait, closed orbits, amplitude-period relation, Jacobi elliptic function.

AMS Subject Classification: $34 \mathrm{C} 05 ; 34 \mathrm{C} 15 ; 34 \mathrm{C} 37 ; 78 \mathrm{~A} 35$.

\section{Introduction}

Consider a uniformly charged ring with a conducting wire placed along the axis of the ring. Assume that a point charge $q$ whose sign is opposite to the sign of the charge $Q$ of the ring, is confined with the wire. Under the assumption that the loss of energy per oscillation due to radiation is negligibly small, the oscillations of this point charge can be modelled by the following scalar nonlinear autonomous differential equation due to A. Beléndez et al. [2]

$$
\frac{d^{2} x}{d t^{2}}+\frac{x}{\left(1+x^{2}\right)^{3 / 2}}=0 .
$$

Copyright (c) 2019 The Author(s). Published by VGTU Press

This is an Open Access article distributed under the terms of the Creative Commons Attribution License (http://creativecommons.org/licenses/by/4.0/), which permits unrestricted use, distribution, and reproduction in any medium, provided the original author and source are credited. 
Since (1.1) has a first integral representing a family of closed orbits surrounding the origin in the phase plane, the initial value problem

$$
x(0)=A, \quad \frac{d x}{d t}(0)=0
$$

has to any $A, 0<A<+\infty$, a unique solution $x(t, A)$ representing a periodic solution with (positive) primitive period $T(A)$. The parameter $A$ can be interpreted as the amplitude of the periodic solution $x(t, A)$. The relation between the amplitude $A$ and the frequency $\omega(A)=2 \pi / T(A)$ is of fundamental interest. In [2], an expression for $\omega(A)$ is presented which contains a wrong factor (see [3]). This incorrect relation has been used in several recent publications $[7,8,9,10]$.

For applications, a key interest is to derive simple approximate frequencyamplitude relations. For this purpose there are a lot of methods, we mention only a few ones: harmonic balance, energy balance, variational iteration and homotopy perturbation. A survey about these methods can be found in [7].

The focus of our paper is not on deriving approximate relations for the dependence of the frequency (period) on the amplitude. We present a study of the mathematical model (1.1) under two different aspects: the qualitative aspect focuses on the determination of the global phase portrait of equation (1.1), the analytical aspect aims to study the dependence of period and frequency of the periodic solutions on the amplitude.

The qualitative investigations show that the global phase portrait of this model consists in the Poincaré disk of a family of closed orbits surrounding the unique finite equilibrium point and of a continuum of homoclinic orbits to the unique equilibrium point at infinity. The local phase portrait of this equilibrium point is investigated by means of the method of desingularization [6] consisting of changes of variables called blow-ups (see chapter 3 in [6]).

The analytical studies provide an expression for the dependence of the frequency of the point charge oscillations on the amplitude. Further, we prove that the period increases monotone with the amplitude, and we determine the growth rate of the primitive period as the amplitude tends to infinity. Finally, we derive a relation between period and amplitude by means of the Jacobi elliptic function $c n$ and the complete elliptic integral of the first kind $K(k)$. For their definition we consider the incomplete elliptic integral of the first kind

$$
u=\int_{0}^{\varphi} \frac{d \theta}{\sqrt{1-k^{2} \sin ^{2} \theta}}=: F(\varphi, k),
$$

where $k$ is called the elliptic module $\left(k^{2}<1\right)$. The Jacobi elliptic functions are defined as inverses of (1.2). Especially, the elliptic cosine $c n u$ is defined by cnu $:=\cos \varphi$, where $\varphi$ is called the amplitude. It follows from (1.2) that the function $c n$ depends also on $k$. In the literature, along the short notation $c n u$ also the full notation $c n(u, k)$ is used. The function $K$ is defined by

$$
K(k):=F(\pi / 2, k)=\int_{0}^{\pi / 2} \frac{d \theta}{\sqrt{1-k^{2} \sin ^{2} \theta}} .
$$




\section{Global phase portrait of the trajectories of equation (1.1) in the Poicaré disk}

For the following we rewrite equation (1.1) as the system

$$
\frac{d x}{d t}=y, \quad \frac{d y}{d t}=-\frac{x}{\left(1+x^{2}\right)^{3 / 2}}
$$

in the $(x, y)$-phase plane. The following properties of system (2.1) can be easily verified.

Lemma 1. The origin is the unique equilibrium point $\mathcal{E}$ of system (2.1) in the finite part of the phase plane.

Lemma 2. System (2.1) has the first integral

$$
H(x, y):=\frac{y^{2}}{2}-\frac{1}{\sqrt{1+x^{2}}}=c, \quad c \geq-1 .
$$

From Lemma 2 we get

Corollary 1. The phase portrait of system (2.1) is symmetric with respect to the $x$-axis as well as with respect to the $y$-axis.

Corollary 2. System (2.1) can be rewritten in the form

$$
\frac{d x}{d t}=\frac{\partial H}{\partial y}, \quad \frac{d y}{d t}=-\frac{\partial H}{\partial x}
$$

that is, (2.1) is a Hamiltonian system.

Lemma 3. The family of orbits $\left\{\mathcal{O}_{c}\right\}$ of system (2.3) defined by

$$
\left\{\mathcal{O}_{c}\right\}:=\left\{(x, y) \in \mathbb{R}^{2}: \quad H(x, y)=c\right\}
$$

consists for $-1<c<0$ of closed orbits located in the finite part of the phase plane. This family of closed orbits has two envelopes, the equilibrium point $\mathcal{E}=\mathcal{O}_{-1}$ and the curve $\mathcal{O}_{0}$ defined by

$$
\mathcal{O}_{0}:=\left\{(x, y) \in \mathbb{R}^{2}: \quad \frac{y^{2}}{2}-\frac{1}{\sqrt{1+x^{2}}}=0\right\} .
$$

For $c>0$, the family $\left\{\mathcal{O}_{c}\right\}$ consists of the curves

$$
\mathcal{O}_{c}:=\left\{(x, y) \in \mathbb{R}^{2}: \quad y= \pm \sqrt{2 c+\frac{2}{\sqrt{1+x^{2}}}}\right\}
$$

where $|y(x)|$ takes its maximum $\sqrt{2(1+c)}$ at $x=0$ and satisfies

$$
\lim _{x \rightarrow \pm \infty}|y(x)|=\sqrt{2 c}
$$

(see Figure 1). 


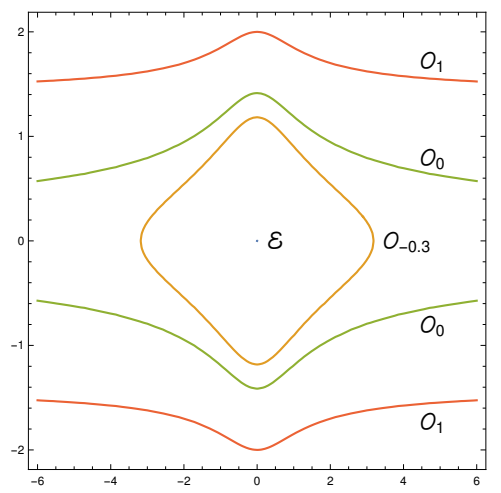

Figure 1. Selected orbits of system (2.1) in a finite part of the phase plane

Lemma 3 implies

Corollary 3. The equilibrium point $\mathcal{E}$ of system (2.1) is a center.

If we consider the velocity $v_{c}(x, y)$ of a point moving along the closed orbit $\mathcal{O}_{c}$ for $-1<c<0$ at the point $\left(x_{c}, 0\right)$, then we get from $(2.1)$

$$
v_{c}^{2}\left(x_{c}, 0\right)=\frac{x_{c}^{2}}{\left(1+x_{c}^{2}\right)^{3}} .
$$

From (2.2) we obtain

$$
x_{c}^{2}=\left(1-c^{2}\right) / c^{2} .
$$

By (2.4) and (2.5) we have

$$
v_{c}^{2}\left(x_{c}, 0\right)=c^{4}\left(1-c^{2}\right) .
$$

Therefore, it holds

$$
v_{c}^{2}\left(x_{c}, 0\right) \rightarrow 0 \quad \text { as } \quad c \rightarrow 0
$$

and we expect that $\mathcal{O}_{0}$ is a heteroclinic cycle. To give an answer to this conjecture we have to study the equilibria of system (2.1) at infinity. For this purpose we apply transformations mapping the phase plane onto planes tangent to the Poincaré sphere at the equator. By means of the transformation

$$
z=1 / y, \quad v=x / y
$$

we can study the existence of equilibria on the equator located at the "ends" of the $y$-axis. We obtain the system

$$
\frac{d z}{d t}=\frac{z^{4} v}{\left(z^{2}+v^{2}\right)^{3 / 2}}, \quad \frac{d v}{d t}=1+\frac{v^{2} z^{3}}{\left(z^{2}+v^{2}\right)^{3 / 2}} .
$$

From (2.6) we get immediately 
Lemma 4. There is no equilibrium point of system (2.1) at infinity located on the equator of the Poincaré sphere at the "ends" of the y-axis.

Using the transformation

$$
z=1 / x, \quad u=y / x
$$

we are looking for equilibria located on the equator of the Poincaré sphere but not located at the "ends" of the $y$-axis. We obtain the system

$$
\frac{d u}{d t}=-\frac{z^{3}}{\left(z^{2}+1\right)^{3 / 2}}-u^{2}, \quad \frac{d z}{d t}=-u z
$$

possessing the unique equilibrium point $u=z=0$, denoted as $\mathcal{E}_{0}$. This equilibrium point is degenerate, also called linearly zero according to [6], since the Jacobi matrix of the right hand side of system $(2.7)$ at $\mathcal{E}_{0}$ is the zero matrix. The following properties of system (2.7) are used to determine the local phase portrait of $\mathcal{E}_{0}$.

\section{Lemma 5.}

(i) System (2.7) is invariant under the transformation $u \rightarrow-u, t \rightarrow-t$, that means that the phase portrait of system (2.7) exhibits a mirror symmetry concerning the z-axis.

(ii) System (2.7) has the invariant straight line $z=0$ which implies that any trajectory starting in the upper (lower) half-plane remains in that half-plane.

(iii) In the half plane $z \geq 0$ we have $d u / d t<0$, except at the origin, where $d u / d t=0$.

(iv) In the interior of the first (second) and of the third (fourth) quadrant of the phase plane of system (2.7) it holds $d z / d t<0(d z / d t>0)$.

(v) The isocline $\mathcal{U}_{0}:=\left\{(u, z) \in \mathbb{R}^{2}: d u / d t=u^{2}+z^{3} /\left(z^{2}+1\right)^{3 / 2}=0\right\}$ exhibits a mirror symmetry concerning the $z$-axis. It consists of two branches located in the lower half-plane forming a singularity at the equilibrium $\mathcal{E}_{0}$ (see the dashed curve in Figure 2).

Lemma 5 implies that any trajectory of system (2.7) starting in the fourth quadrant of the phase plane has $\mathcal{E}_{0}$ as $\omega$-limit point. According to the mirror symmetry with respect to the $z$-axis, any trajectory of system (2.7) in the third quadrant has $\mathcal{E}_{0}$ as $\alpha$-limit point. Thus, the half-plane $z<0$ is filled with homoclinic orbits to the equilibrium point $\mathcal{E}_{0}$ (see Figure 2).

The phase portrait of system (2.7) in the upper half-plane is characterized by the mirror symmetry with respect to the $z$-axis and by the properties $d u / d t<0$. Additionally, in the first quadrant it holds $d z / d t<0$. Thus, any trajectory of system (2.7) in the first quadrant either crosses the $z$-axis or it possesses $\mathcal{E}_{0}$ as $\omega$-limit point. Hence, any trajectory crossing the $z$-axis has a minimum at the $z$-axis (see Figure 3). 


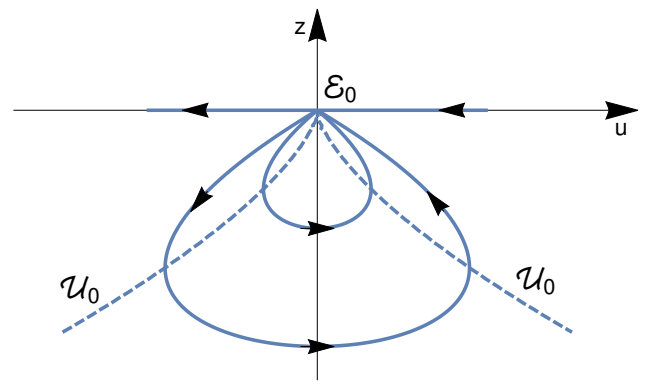

Figure 2. Phase portrait of system (2.1) in the lower half-plane

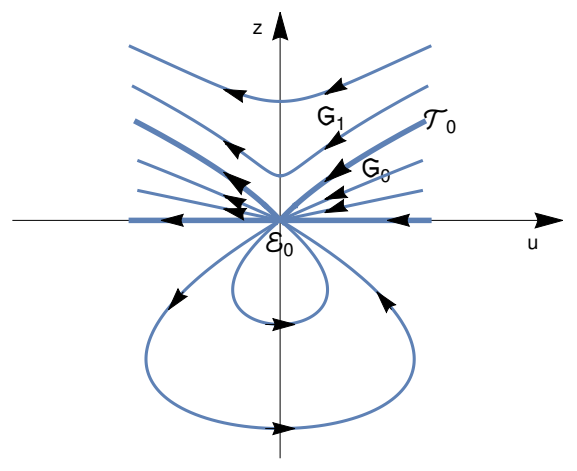

Figure 3. Phase portrait of system (2.1) in the upper half-plane

The interesting question concerns the existence of a trajectory $\mathcal{T}_{0}$ in the first quadrant having $\mathcal{E}_{0}$ as $\omega$-limit set and separating the regions $\mathcal{G}_{0}$ and $\mathcal{G}_{1}$ such that all trajectories starting in $\mathcal{G}_{0}$ have $\mathcal{E}_{0}$ as $\omega$-limit set, while all trajectories starting in $\mathcal{G}_{1}$ cross the $z$-axis (see Figure 3 ). To answer this question we apply the method of blowing up the equilibrium point $\mathcal{E}_{0}$ by means of the transformation $\mathcal{H}$ :

$$
w=z, \quad v=u / z .
$$

We obtain from (2.7) and (2.8) the system

$$
\frac{d v}{d t}=-\frac{w^{2}}{\left(1+w^{2}\right)^{3 / 2}}, \quad \frac{d w}{d t}=-v w^{2} .
$$

System (2.9) is invariant under the transformation $v \rightarrow-v, t \rightarrow-t$, that means its phase portrait possesses the mirror symmetry with respect to the $w$-axis. Moreover, this system exhibits the peculiarity that the invariant straight line $w=0$ consists of equilibria. That means the degenerate equilibrium $\mathcal{E}_{0}$ of system (2.7) has been blown up to a line of equilibria in system (2.9). Additionally, system (2.9) has the first integral

$$
\frac{v^{2}}{2}-\frac{w}{\sqrt{1+w^{2}}}=c .
$$


The phase portrait of system (2.9) in the upper half-plane is represented in Figure 4.

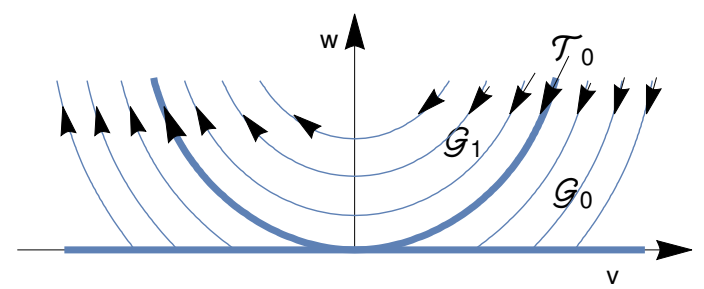

Figure 4. Phase portrait of system (2.9) in the upper half-plane

There exists exactly one trajectory of system (2.9) starting in the first quadrant which has the origin as $\omega$-limit point and which separates the regions $\mathcal{G}_{0}$ and $\mathcal{G}_{1}$ (see Figure 4 ) such that to each trajectory starting in the region $\mathcal{G}_{0}$ there exists a unique point on the $v$-axis which is the $\omega$-limit point of this trajectory. Using the inverse of the transformation $\mathcal{H}$ we get that all these trajectories corresponds to trajectories of system (2.7) having $\mathcal{E}_{0}$ as $\omega$-limit point. Thus, the local phase portrait belonging to the equilibrium point $\mathcal{E}_{0}$ of system (2.7) looks as represented in Figure 5.

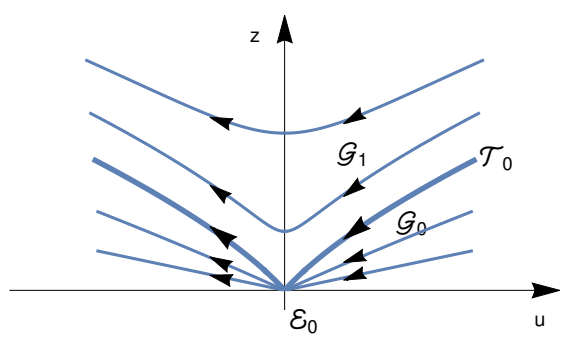

Figure 5. Local phase portrait of system (2.9) near $\mathcal{E}_{0}$

Taking into account our qualitative results described above we get the following result:

Theorem 1. The orbits $\left\{\mathcal{O}_{c}\right\}$ of system (2.3) defined for $c \geq-1$ represent in the Poincaré disk

- for $c=-1$ the equilibrium point $\mathcal{E}$ at the origin,

- for $-1<c<0$ closed orbits with finite primitive period,

- for $c \geq 0$ homoclinic orbits to the unique equilibrium point $\mathcal{E}_{0}$ at infinity.

The corresponding global phase portrait of system (2.1) in the Poincaré disk is represented in Figure 6 . We note that the points $\mathcal{E}_{-1}$ and $\mathcal{E}_{+1}$ coincide and represent the equilibrium point $\mathcal{E}_{0}$.

In the following section we study the closed orbits of the family $\mathcal{O}_{c}$ which are periodic solutions. Especially, we are interested in the dependence of the primitive period on the amplitude. 


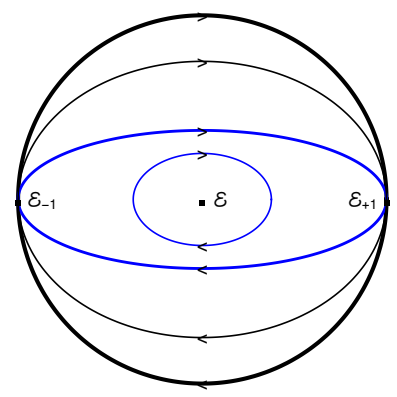

Figure 6. Global phase portrait of system (2.1) in the Poincaré disk

\section{Periodic orbits and their period}

We denote by $\Gamma_{A}$ the orbit of system (2.1) passing the point $(x=A, y=$ $0)$. Without loss of generality we may assume $A \geq 0$. By (2.2), $\Gamma_{A}$ has the representation

$$
\Gamma_{A}:=\left\{(x, y) \in \mathbb{R}^{2}: \quad \frac{y^{2}}{2}-\frac{1}{\sqrt{1+x^{2}}}+\frac{1}{\sqrt{1+A^{2}}}=0\right\} .
$$

From (3.1) it follows that $\Gamma_{A}$ is a closed orbit for any $A \geq 0$. Since the parameter $A$ can be interpreted as the amplitude of the closed orbit $\Gamma_{A}$, we can conclude that the point charge oscillator (2.1) has to any amplitude $A$ a unique periodic solution. But from the property that the function $x /\left(1+x^{2}\right)^{3 / 2}$ arising in equation (1.1) does not tend to $+\infty$ as $|x|$ tends to $+\infty$, we cannot conclude that the family of closed orbits $\left\{\Gamma_{A}\right\}_{A \geq 0}$ covers the full phase plane.

For the sequel we denote by $T(A)$ the (positive) primitive period of $\Gamma_{A}$ for $A>0$. By the symmetry properties of the closed orbit $\Gamma_{A}$, for the determination of $T(A)$ it is sufficient to calculate the time for running along the part of $\Gamma_{A}$ located in the first quadrant. Using the relation $d t=d x / y$ in (2.1) and the representation of $\Gamma_{A}$ in the first quadrant

$$
\Gamma_{A}:=\left\{(x, y) \in \mathbb{R}^{+} \times \mathbb{R}^{+}: \quad y=\sqrt{2} \sqrt{\frac{1}{\sqrt{1+x^{2}}}-\frac{1}{\sqrt{1+A^{2}}}}\right\},
$$

which follows from (3.1), we get

\section{Lemma 6.}

$$
T(A)=\frac{4}{\sqrt{2}} \int_{0}^{A} \frac{d x}{\left(\frac{1}{\sqrt{1+x^{2}}}-\frac{1}{\sqrt{1+A^{2}}}\right)^{0.5}}=2 \sqrt{2} A \int_{0}^{1} \frac{d s}{\left(\frac{1}{\sqrt{1+A^{2} s^{2}}}-\frac{1}{\sqrt{1+A^{2}}}\right)^{0.5}} .
$$

We note that this relation corrects a corresponding expression due to A. Beléndez et al. in [2] (see [3]) which has been used in the recent papers $[7,8,9,10]$.

In what follows we study the dependence of the period $T(A)$ on the amplitude $A$. First we ask the question whether $T(A)$ increases monotone with $A$. 
To answer this question we introduce the functions

$$
V(x):=1-\frac{1}{\sqrt{1+x^{2}}}, \quad H_{1}(x, y):=\frac{y^{2}}{2}+V(x)=H(x, y)+1 .
$$

$V$ satisfies

$$
V(0)=0, V^{\prime}(0)=0, V^{\prime \prime}(0)>0,0<V(x)<1 \quad \text { for } \quad x \neq 0 .
$$

Thus, $H_{1}(x, y)$ is a Hamiltonian for system (2.1) with a nondegenerate minimum at the origin. The corresponding level energy curves with energy $E$ are defined for $E \in(0,1)$. Let $K(E):=\{x \in \mathbb{R}: V(x) \leq E\}$. Additionally we introduce the function

$$
W(x):=V(x) /\left(V^{\prime}(x)\right)^{2} .
$$

By a result due to $\mathrm{C}$. Chicone [5], the function $T(A)$ is monotone increasing if $W^{\prime \prime}(x)$ is positive on $(0,1)$.

According to (3.3) and (3.4) we have for $x \neq 0$

$$
\begin{aligned}
W^{\prime \prime}(x) & =\frac{-3}{x}+\frac{3}{1+x}+\frac{3 V(x)}{\sqrt{1+x}}-\frac{9 \sqrt{1+x} V(x)}{x}+\frac{6(\sqrt{1+x})^{3} V(x)}{x} \\
& =2 x V(x)>0 .
\end{aligned}
$$

Thus it holds:

Theorem 2. The period $T(A)$ increases monotone with the amplitude $A$.

From Theorem 2 we get

Corollary 4. The origin is not an isochronous center of system (2.1).

Next we derive an asymptotic estimate of the growth rate of $T(A)$ as $A$ tends to $+\infty$. Taking into account the relations

$$
\int_{0}^{1} \frac{d s}{\left(\frac{1}{\sqrt{1+A^{2} s^{2}}}-\frac{1}{\sqrt{1+A^{2}}}\right)^{0.5}}=\sqrt{A} \int_{0}^{1} \frac{d s}{\left(\frac{1}{\sqrt{1 / A^{2}+s^{2}}}-\frac{1}{\sqrt{1 / A^{2}+1}}\right)^{0.5}}
$$

and

$$
\lim _{A \rightarrow+\infty} \int_{0}^{1} \frac{d s}{\left(\frac{1}{\sqrt{1 / a^{2}+s^{2}}}-\frac{1}{\sqrt{1 / A^{2}+1}}\right)^{0.5}}=\int_{0}^{1} \sqrt{\frac{s}{1-s}} d s=2
$$

we obtain from (3.2) the result:

Lemma 7. The primitive period $T(A)$ satisfies the relation

$$
T(A)=O(A \sqrt{A}) \text { as } A \rightarrow+\infty .
$$


Another expression for the period $T(A)$ can be obtained as follows. We denote by $\Gamma^{B}$ the trajectory of system $(2.1)$ passing the point $(0, B)$. Without loss of generality we may assume $B \geq 0$. From (2.2) we obtain for $\Gamma^{B}$ the representation

$$
\Gamma^{B}:=\left\{(x, y) \in \mathbb{R}^{2}: \quad \frac{y^{2}}{2}-\frac{1}{\sqrt{1+x^{2}}}+1-\frac{B^{2}}{2}=0\right\} .
$$

The expression (3.5) implies that $\Gamma^{B}$ is a closed orbit as long as $B$ satisfies $0<B^{2}<2$. Since the parameter $B$ can be interpreted as the maximum velocity on the closed orbit $\Gamma^{B}$, it holds:

Lemma 8. $\sqrt{2}$ is an upper bound for the maximum velocity on all periodic solutions of (2.1).

Taking into account that the closed orbit $\Gamma_{A}$ intersects the positive $y$-axis at the point $\left(0, \sqrt{2\left(1-1 / \sqrt{1+A^{2}}\right)}\right)$, we can conclude that the closed orbits $\Gamma_{A}$ and $\Gamma^{B}$ coincide if it holds

$$
B=\tilde{B}(A):=\sqrt{2\left(1-1 / \sqrt{1+A^{2}}\right)} \text { for } A>0,
$$

which is equivalent to

$$
A=\tilde{A}(B):=\frac{\sqrt{4-\left(2-B^{2}\right)^{2}}}{2-B^{2}} \text { for } 1<B^{2}<2 .
$$

For the determination of the (positive) primitive period $\tau(B)$ of $\Gamma^{B}$ we can restrict ourselves to the part of $\Gamma^{B}$ located in the first quadrant. For its representation we use the relation

$$
x=\frac{\sqrt{4-\left(y^{2}-B^{2}+2\right)^{2}}}{y^{2}-B^{2}+2}=: f(y, B)
$$

which follows from (3.5). According to (2.1) and (3.7) we have

$$
-\frac{\left(1+f(y, B)^{2}\right)^{3 / 2}}{f(y, B)} d y=d t .
$$

Taking into account the relation

$$
\frac{\left(1+f(y, B)^{2}\right)^{3 / 2}}{f(y, B)}=\frac{8}{\left(y^{2}-B^{2}+2\right)^{2} \sqrt{4-\left(y^{2}-b^{2}+2\right)^{2}}}
$$

we get from $(3.8)$

$$
\tau(B)=32 B \int_{0}^{1} \frac{d s}{\left(y^{2}-B^{2}+2\right)^{2} \sqrt{4-\left(B^{2} s^{2}-B^{2}+2\right)^{2}}} .
$$

The advantage of this representation of the period of the closed curves of system (2.1) consists in the fact that the right hand side of (3.9) is an elliptic integral. In the following section we derive an expression for $\tau(B)$ and $T(A)$ by means of the complete elliptic integral of the first kind $K(k)$ and the Jacobi elliptic function $\mathrm{cn}$. 


\section{Analytic relation between amplitude and period of the point charge oscillations by using Jacobi's elliptic function $c n$}

The expression (3.9) for $\tau(B)$ can be rewritten in the form

$$
\begin{aligned}
\tau(B) & =32 B \int_{0}^{1} \frac{d s}{\left(B^{2} s^{2}-B^{2}+2\right)^{2} \sqrt{4-\left(B^{2} s^{2}-B^{2}+2\right)^{2}}} \\
& =\frac{32}{B^{5}} \int_{0}^{1} \frac{d s}{\left(s^{2}+\frac{2-B^{2}}{B^{2}}\right)^{2} \sqrt{\left(\frac{4-B^{2}}{B^{2}}+s^{2}\right)\left(1-s^{2}\right)}} .
\end{aligned}
$$

In [4] we find on page 49 (see relation (213.13)) the formula

$$
\int_{\gamma}^{\beta} \frac{R\left(s^{2}\right) d s}{\sqrt{\left.\alpha^{2}+s^{2}\right)\left(\beta^{2}-s^{2}\right)}}=g \int_{0}^{u_{1}} R\left(\beta^{2} c n^{2} u\right) d u,
$$

where $R$ is any rational function, $c n$ is one of the three Jacobi's elliptic functions sn, $c n$ and $d n, \beta$ and $\gamma$ are constants satisfying $\beta>\gamma \geq 0, g$ and $u_{1}$ are defined by the relations

$$
g=\frac{1}{\sqrt{\alpha^{2}+\beta^{2}}}, \quad u_{1}=F(\varphi, k)=\int_{0}^{\varphi} \frac{d \theta}{\sqrt{1-k^{2} \sin ^{2} \theta}}
$$

where

$$
\varphi=\arccos (\gamma / \beta), \quad k^{2}=\frac{\beta^{2}}{\alpha^{2}+\beta^{2}}<1
$$

It is clear that the integral in (4.1) is a special case of the integral (4.2). From (4.1)-(4.3) we get

$$
\begin{array}{r}
\gamma=0, \quad \beta=1, \quad \alpha^{2}=\frac{4-B^{2}}{B^{2}}, \quad R\left(s^{2}\right)=\frac{1}{\left(s^{2}+\frac{2-B^{2}}{B^{2}}\right)^{2}}, \\
g=\frac{B}{\sqrt{4-B^{2}}}, \quad k^{2}=\frac{B^{2}}{4}, \quad \varphi=\arccos 0=\frac{\pi}{2}, \quad u_{1}=F\left(\frac{\pi}{2}, k\right),
\end{array}
$$

where $F\left(\frac{\pi}{2}, k\right)$ coincides with the complete elliptic integral of the first kind $K(k)$ introduced in (1.3). From (4.1), (4.2) and (4.4) we obtain the representation

$$
\tau(B)=\frac{32}{B^{4} \sqrt{4-B^{2}}} \int_{0}^{K\left(\frac{B}{2}\right)} \frac{d u}{\left(c n^{2} u+\frac{2-B^{2}}{B^{2}}\right)^{2}} .
$$

As we noted in the introduction, the Jacobi elliptic functions depend on the parameter $k, k^{2}<1$. If we use the full notion $c n(u, k)$, then in our case by (4.4) and Lemma 8 it holds $k^{2}=B^{2} / 4<1 / 2$. Taking into account this remark and using the relation (3.6), we arrive at the result: 
Theorem 3. To given $A, 0<A<+\infty$, the closed orbit $\Gamma_{A}$ with the amplitude $A$ has the primitive period

$$
T(A)=\frac{32}{(\tilde{B}(A))^{4} \sqrt{4-(\tilde{B}(A))^{2}}} \int_{0}^{K\left(\frac{\tilde{B}(A)}{2}\right)} \frac{d u}{\left(c n^{2}(u, \tilde{B}(A) / 2)+\frac{2-(\tilde{B}(A))^{2}}{(\tilde{B}(A))^{2}}\right)^{2}},
$$

where the functions $\tilde{B}$ and $K$ are defined in (3.6) and (1.3), respectively.

Remark 1. If we plot the functions for $T(A)$ defined in (3.2) and (4.5) respectively, we get the same picture.

Remark 2. We emphasize that the relation (4.5) is valid for any amplitude $A$. Under the condition $A<1$, A. Beléndez et al. derived in [1] by means of the method of first order harmonic balance an approximate relation for $T(A)$ which also depends on $K(A)$.

\section{Conclusions}

The global phase portrait of system (2.1) implies that the closed orbits describing the point charge oscillations tend to a homoclinic orbit as the amplitude $A$ tends to infinity. Thus, the corresponding period $T(A)$ tends also to infinity. By means of analytical investigations we derive an expression for the dependence of the period on the amplitude, prove that the period increases monotone with the amplitude and determine the growth rate of $T(A)$ as $A$ tends to $+\infty$. Finally, we derive a relation between the period $T(A)$ and the corresponding amplitude $A$ by means of the complete elliptic integral of the first kind $K(k)$ and the Jacobi elliptic function $c n$ which holds for any $A$.

\section{References}

[1] A. Beléndez, E. Arribas and J. Francés. Notes on "Application of the Hamiltonian approach to nonlinear oscillators with rational and irrational elastic terms". Mathematical and Computer Modeling, 54(11-12):3204-3209, 2011. https://doi.org/10.1016/j.mcm.2011.06.024.

[2] A. Beléndez, E. Fernández, J.J. Rodes, R. Fuentes and I. Pascual. Harmonic balancing to nonlinear oscillations of a punctual charge in the electric field of charged ring. Physics Letters A, 373(7):735-740, 2009. https://doi.org/10.1016/j.physleta.2008.12.042.

[3] A. Beléndez and K.R. Schneider. Erratum to "Harmonic balancing to nonlinear oscillations of a punctual charge in the electric field of charged ring" [Phys. Lett. A 373(7)(2009) 735-740]. Physics Letters A, 383(11):1214, 2019. https://doi.org/10.1016/j.physleta.2019.02.018.

[4] P.F. Byrd and M.D. Friedman. Handbook of elliptic integrals for engineers and physicists, volume 67 of Grundlehren der Mathematischen Wissenschaften. Springer-Verlag New-York-Heidelberg, 1971. 
[5] C. Chicone. The monotonicity of the period function for planar Hamiltonian vector fields. J. Differential Equations, 69(3):310-321, 1987. https://doi.org/10.1016/0022-0396(87)90122-7.

[6] F. Dumortier, J. Llibre and J.C. Artes. Qualitative theory of planar differential systems. Universitext, Springer, Berlin, 2006.

[7] O. Gonzáles-Gaxiola, G. Chacón-Acosta and J.A. Santiago. Nonlinear oscillations of a point charge in the electric field of charged ring using a particular He's frequency-amplitude formulation. International Journal Applied Computational Mathematics, 4:43, 2018. https://doi.org/10.1007/s40819-017-0479-1.

[8] S. Valipour, R. Fallahpour, M.M. Moridani and S. Chakouvari. Nonlinear dynamic analysis of a punctual charge in the electric field of a charged ring via a modified frequency-amplitude formulation. Propulsion and Power Research, 5(1):81-86, 2016. https://doi.org/10.1016/j.jppr.2016.01.001.

[9] M.K. Yazdi. Corrigendum to "Analysis of nonlinear oscillations of a punctual charge in the electrical field of a charged ring via a Hamiltonian approach and the energy balance method" [Computers and mathematics with applications 62:486490 (2011)]. Computers and Mathematics with Applications, 62(6):2681-2682, 2011. https://doi.org/10.1016/j.camwa.2011.08.006.

[10] A. Yildirim, H. Ascari, Z. Saadatnia, M. Kalami Yazdi and Y. Khan. Analysis of nonlinear oscillations of a punctual charge in the electrical field of a charged ring via a Hamiltonian approach and the energy balance method. Computers and Mathematics with Applications, 62(1):486-490, 2011. https://doi.org/10.1016/j.camwa.2011.05.029. 